\title{
A reemergência da coqueluche em países desenvolvidos: um problema também para o Brasil?
}

\author{
The resurgence of pertussis in developed \\ countries: a problem for Brazil as well?
}

Paula Mendes Luz 1,2

Cláudia Torres Codeço 1

Guilherme Loureiro Werneck 2,3

\footnotetext{
1 Programa de Computação Científica, Fundação Oswaldo Cruz. Av. Brasil 4365, Rio de Janeiro, $R J$ 21045-900, Brasil. pluz@fiocruz.br

2 Instituto de Medicina Social, Universidade do Estado do Rio de Janeiro. Rua São Francisco Xavier 524, 7o andar, Bloco D, Rio de Janeiro, $R J$ 20559-900, Brasil.

3 Núcleo de Estudos de Saúde Coletiva, Universidade Federal do Rio de Janeiro. Cidade Universitária, Bloco K, C. P. 68037 , Rio de Janeiro, $R J$ 21941-590, Brasil.
}

\begin{abstract}
Pertussis is currently considered an important public health problem in developed countries. In most of these countries, mass immunization for pertussis was initiated in the $1950 \mathrm{~s}$ and was followed by a marked decrease in disease incidence. In the 1970s, pertussis was apparently under control in countries were vaccine coverage was maintained high. However, in the last two decades of the 20th century, the number of reported cases increased in all age groups, including adolescents and adults, indicating resurgence of the disease. This brief note aims to present the possible reasons for resurgence of this disease and to discuss the prospects of its future dynamics in Brazil. There has been no evidence to date for the resurgence of pertussis in Brazil. However, since mass immunization in Brazil began only in the 1980s, one cannot rule out the possibility that pertussis will resurge in the near future. Therefore, it is important that public health services closely monitor the epidemiological situation of pertussis in order, if necessary, to rapidly update the current immunization strategy.
\end{abstract}

Key words Whooping Cough; Emerging Communicable Diseases; Diphtheria-Tetanus-Pertussis Vaccine; Vaccine Coverage

Resumo Atualmente, a coqueluche representa um crescente problema de saúde pública em países desenvolvidos. Nesses países, a imunização em massa de crianças, iniciada na década de 50, foi seguida por uma brusca diminuição da incidência da doença. Assim, nos anos 70, a coqueluche não representava uma importante ameaça à saúde destas populações. Porém, nas décadas de 80 e 90, ocorreu um aumento do número de casos em todas as faixas etárias, incluindo adolescentes e adultos, indicando a reemergência da coqueluche. Nesta nota objetiva-se apresentar as possíveis causas para esta reemergência e discutir perspectivas para a dinâmica da doença no Brasil. No momento, ainda não existem evidências de reemergência da coqueluche no país. No entanto, como a vacinação em massa no Brasil foi iniciada apenas na década de 80, não se pode descartar a hipótese de uma futura reemergência da doença. Assim, é importante que serviços de vigilância e controle de doenças monitorem a situação epidemiológica da coqueluche para que, se necessário, estejam preparados para reformular as estratégias de imunização.

Palavras-chave Coqueluche; Doenças Transmissíveis Emergentes; Vacina contra Difteria-Tétano-Pertussis; Cobertura Vacinal 


\section{Introdução}

A coqueluche, doença infecciosa aguda, tendo como principal agente etiológico a bactéria Bordetella pertussis, é causa importante de morbi-mortalidade infantil. Estima-se que cerca de 50 milhões de casos e 300 mil óbitos ocorram a cada ano no mundo, e a letalidade em crianças, pode aproximar-se de $4 \%$ (WHO, 2001). Mundialmente, a coqueluche é a terceira causa de morte entre as doenças imunopreviníveis (Ivanoff \& Robertson, 1997).

Atualmente, países desenvolvidos deparamse com a reemergência da coqueluche (Das, 2002). Nesses países, a imunização em massa de crianças com a vacina DPT celular (contra difteria, coqueluche e tétano) reduziu a incidência e mortalidade entre crianças até quatro anos de idade. Visto que a imunidade adquirida artificialmente não é duradoura, as altas taxas de cobertura vacinal determinaram uma mudança no padrão da infecção. Hoje em dia, além de atingir as crianças ainda não completamente imunizadas, a coqueluche afeta também as crianças vacinadas maiores de quatro anos de idade, adolescentes e adultos (Edwards et al., 1999).

Esta situação tem mobilizado a comunidade acadêmica internacional e diversos fóruns têm sido realizados com o intuito de discutir as possíveis causas para esta reemergência, as prováveis situações futuras e a melhor conduta preventiva a ser adotada. Nesta nota, apresentamos as possíveis causas para a reemergência da coqueluche e discutimos perspectivas para a dinâmica da doença em nosso meio.

\section{Dinâmica da coqueluche nos países desenvolvidos}

Na era pré-vacinal, a incidência anual da coqueluche na Inglaterra e países nórdicos era de 200 a 1.000 casos por 100 mil indivíduos (Ivanoff \& Robertson, 1997). Em grande parte dos países desenvolvidos, a imunização em massa de crianças, iniciada na década de 50, foi seguida por uma brusca diminuição da incidência da doença. Durante a década de 70, entretanto, uma série de críticas à vacina DTP celular determinou um declínio na cobertura vacinal na Inglaterra, Suécia e Japão (Ivanoff \& Robertson, 1997). Nesses países, a incidência da coqueluche aumentou rapidamente para mais de 100 casos por 100 mil indivíduos. Por outro lado, países que mantiveram a taxa de cobertura vacinal acima de $90 \%$, para as três doses da vacina DPT, notificaram menos de 10 casos por 100 mil indivíduos (Ivanoff \& Robertson, 1997). Atualmente, nos Estados Unidos, Inglaterra e Canadá, a cobertura vacinal continua alta e estudos estimam que a incidência real da doença esteja em torno de 400 casos por $100 \mathrm{mil}$ indivíduos (Campins-Marti et al., 2001; Strebel et al., 2001).

A distribuição etária dos casos foi modificada após a introdução da vacinação em massa de crianças. Na era pré-vacinal, o valor máximo da incidência ocorria em menores de seis anos de idade (Edwards et al., 1999). Por outro lado, a era pós-vacinal caracterizou-se por uma alta proporção de casos em menores de seis meses, ou seja, crianças ainda não completamente imunizadas (Farizo et al., 1992). Países onde ocorreu um decréscimo da cobertura vacinal nos anos 70, apresentaram na época, uma alta incidência entre crianças de 1 a 6 anos de idade. Nas décadas de 1980 e 1990, diversos estudos mostraram um aumento do número de casos entre adolescentes e adultos (Guris et al., 1999; Juretzko et al., 2001; Mink et al., 1992). Atualmente, a incidência da coqueluche na maioria dos países desenvolvidos apresenta crescimento em todas as faixas etárias (Campins-Marti et al., 2001).

O aumento do número de casos de coqueluche entre adultos promove uma série de repercussões indesejáveis (Campins-Marti et al., 2001). Um estudo conduzido na Alemanha, por exemplo, observou que aproximadamente $80 \%$ dos adultos com coqueluche apresentavam tosse por mais de três semanas, metade deles relatavam vômitos, $25 \%$ sofreram algum tipo de complicação (otite média, pneumonia, perda de peso e fratura de costela) e broncoscopias foram realizadas desnecessariamente (PostelsMultani et al., 1995). Adicionalmente, em pacientes idosos, a coqueluche pode ser fatal (Mertens et al., 1999).

\section{Hipóteses explicativas \\ para a reemergência da doença em países desenvolvidos}

Diversas hipóteses têm sido sugeridas para explicar a reemergência da coqueluche em países desenvolvidos, dentre elas destacam-se:

- Perda de imunidade: a perda da imunidade adquirida por meio da vacina, na ausência de reforços vacinais, após 5 a 10 anos, seria suficiente para explicar a ocorrência da doença entre adolescentes e adultos. Isto ocorre porque a vacinação em massa de crianças determina uma situação em que a circulação da bactéria entre os indivíduos da comunidade torna-se 
restrita e, consequentemente, deixam de ocorrer os reforços naturais (Edmunds et al., 2002). Sem os reforços naturais, a imunidade adquirida é perdida com o tempo, levando indivíduos a retornarem paulatinamente para a condição de suscetíveis.

- Novos métodos diagnósticos: a utilização de melhores métodos para o diagnóstico da coqueluche (como a reação da cadeia da polimerase - PCR) não pode ser descartada como explicação plausível para o aumento do número de casos de coqueluche. A "reemergência”, neste caso, resultaria apenas da identificação de uma dinâmica subjacente não observada previamente (Skowronski et al., 2002).

- Melhoria dos sistemas de vigilância epidemiológica: esta hipótese sugere que a reemergência deve-se a uma maior capacidade de detecção de casos pelos sistemas de vigilância epidemiológica.

- Redução da eficácia vacinal: no Canadá, foi observada uma baixa eficácia vacinal de lotes de vacinas utilizados no passado, gerando um efeito coorte (Ntezayabo et al., 2003).

- Evolução do patógeno: pesquisadores holandeses sustentam que vacinação em massa de crianças, praticada há mais de cinqüenta anos, forçou a evolução de uma bactéria não mais prevenida pela vacina (Mooi et al., 2001). Desta forma, o que hoje estaríamos observando seria o surgimento de uma nova forma de coqueluche.
De qualquer forma, estas diversas hipóteses não são necessariamente excludentes e uma combinação delas poderia estar operando para promover a reemergência da coqueluche (Guris et al., 1999).

\section{E no Brasil?}

No Brasil, com base nos dados de notificação compulsória, não existem, ainda, sinais de reemergência da coqueluche. A vacinação sistemática das crianças brasileiras foi iniciada em 1983, aproximadamente trinta anos após o início da vacinação nos países de economia central. Desde então, a dinâmica observada é a de diminuição do número de casos notificados em resposta ao aumento da cobertura vacinal (Figura 1) (Waldman et al., 1999). As crianças menores de um ano são as mais acometidas pela doença, em seguida estão as de 1 a 4 anos e, por último, as crianças de 5 a 9 anos. Os adultos correspondem a apenas 2 a $3 \%$ dos casos (FUNASA, 2002).

Esta disparidade entre a dinâmica da coqueluche em países desenvolvidos e no Brasil suscita a seguinte questão: será que no nosso meio a coqueluche está sob controle, como parecem indicar os dados de notificação, ou existe uma gradual modificação na dinâmica da transmissão, que não tem sido detectada pelos sistemas de vigilância epidemiológica?

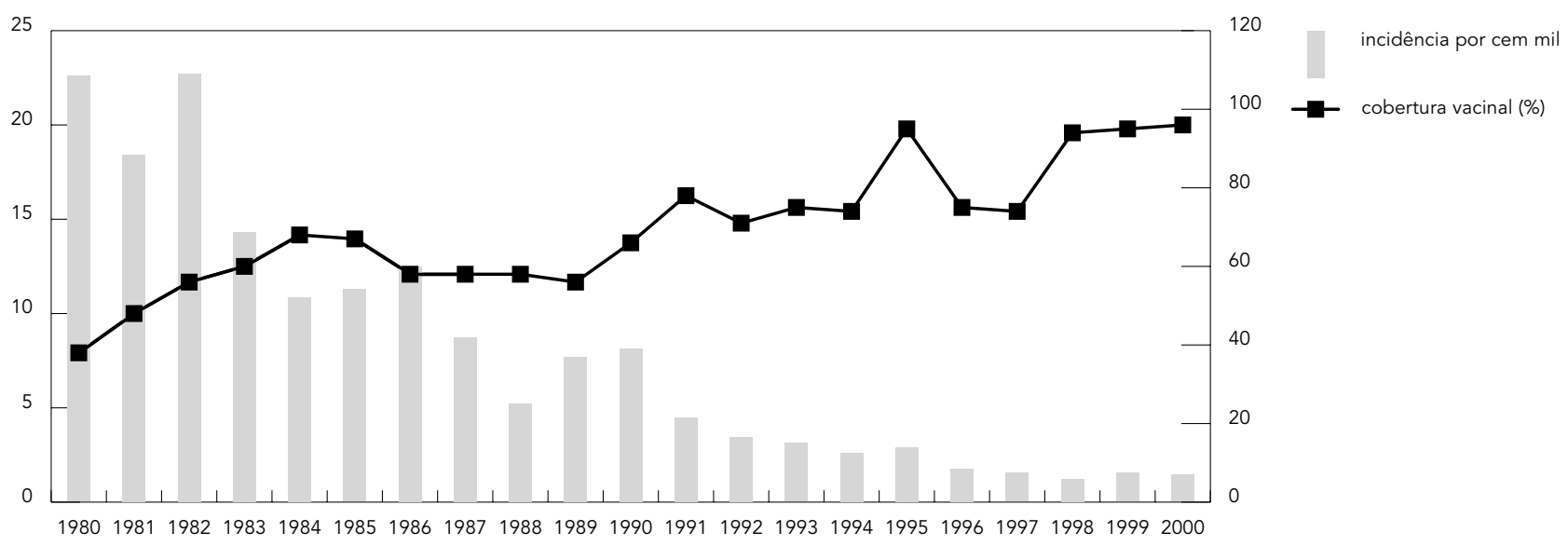

Fonte: Dados obtidos nas home-pages da Fundação Nacional de Saúde <http://www.funasa.gov.br> e do DATASUS <http://www.datasus.gov.br> 
Não existem estudos no Brasil que possibilitem responder inequivocamente se há ou não reemergência da coqueluche em nosso meio. Ainda assim, algumas hipóteses podem ser levantadas. Primeiro, é possível que a coqueluche esteja de fato reemergindo no Brasil, mas não de forma perceptível. Nos países onde notou-se a reemergência, uma crescente atividade da doença é observada em adultos, sendo que a maioria dos casos é de oligossintomáticos. Apenas com a conscientização dos profissionais de saúde sobre a possível ocorrência da coqueluche em adultos, poder-se-ia esperar a identificação desses casos. Por outro lado, um aumento da atividade da doença em adultos geralmente incorre num aumento da incidência em crianças, algo que não se tem observado no Brasil. Este dado apóia a noção de que a coqueluche não estaria, de fato, reemergindo no país.

Alternativamente, é possível que a reemergência da coqueluche ainda venha a ser observada no Brasil. Na maioria dos países desenvolvidos a vacinação em massa iniciou com cerca de trinta anos de antecedência em relação ao Brasil. É razoável supor que a reemergência da coqueluche seja um fenômeno ainda não observado por aqui, simplesmente porque a dinâmica hospedeiro-parasita vigente nos países desenvolvidos não está sincronizada com aquela observada no Brasil. Neste caso, não se pode descartar a hipótese de que a reemergência da coqueluche venha ser uma realidade para o nosso país.

Atualmente, encontram-se disponíveis novas alternativas para a prevenção da coqueluche, como a vacina DPT acelular, mais tolerada por adultos (Edwards et al., 1999). Essas alternativas possibilitam a implementação de novas estratégias vacinais possivelmente mais apropriadas para situações de reemergência da doença. Conseqüentemente, é fundamental que os serviços de vigilância e controle de doenças brasileiros monitorem a situação epidemiológica da coqueluche para que, se necessário, estejam preparados para reformular suas estratégias de imunização.

\section{Referências}

CAMPINS-MARTI, M.; CHENG, H. K.; FORSYTH, K.; GUISO, N.; HALPERIN, S.; HUANG, L. M.; MERTSOLA, J.; OSELKA, G.; WARD, J.; WIRSING-VON KONIG, C. H. \& ZEPP, F., 2001. Recommendations are needed for adolescent and adult pertussis immunisation: Rationale and strategies for consideration. Vaccine, 20:641-646.

DAS, P., 2002. Whooping cough makes global comeback. Lancet Infectious Diseases, 2:322.

EDMUNDS, W. J.; BRISSON, M.; MELEGARO, A. \& GAY, N. J., 2002. The potential cost-effectiveness of acellular pertussis booster vaccination in England and Wales. Vaccine, 20:1316-1330.

EDWARDS, K. M.; DECKER, M. D. \& MORTIMER, E. A., 1999. Pertussis vaccine. In: Vaccines (S. A. Plotkin \&W. A. Orenstein, ed.), pp. 293-337, Philadelphia: W. B. Saunders. 
FARIZO, K. M.; COCHI, S. L.; ZELL, E. R.; BRINK, E. W.; WASSILAK, S. G. \& PATRIARCA, P. A., 1992. Epidemiological features of pertussis in the United States, 1980-1989. Clinical Infectious Diseases, 14:708-719.

FUNASA (Fundação Nacional de Saúde), 2002. Guia de Doenças. 20 Setembro 2002 <http://www.funasa.gov.br>.

GURIS, D.; STREBEL, P. M.; BARDENHEIER, B.; BRENNAN, M.; TACHDJIAN, R.; FINCH, E.; WHARTON, M. \& LIVENGOOD, J. R., 1999. Changing epidemiology of pertussis in the United States: Increasing reported incidence among adolescents and adults, 1990-1996. Clinical Infectious Diseases, 28:12301237.

IVANOFF, B. \& ROBERTSON, S. E., 1997. Pertussis Vaccine Trials. Pertussis: A worldwide problem. Developments in Biological Standardization, 89:3-13.

JURETZKO, P.; FABIAN-MARX, T.; HAASTERT, B.; GIANI, G.; VON KRIES, R. \& WIRSING-VON KONIG, C. H., 2001. Pertussis in Germany: Regional differences in management and vaccination status of hospitalized cases. Epidemiology and Infection, 127:63-71.

MERTENS, P. L.; STALS, F. S.; SCHELLEKENS, J. F.; HOUBEN, A. W. \& HUISMAN, J., 1999. An epidemic of pertussis among elderly people in a religious institution in The Netherlands. European Journal of Clinical Microbiology and Infectious Disease, 18:242-247.

MINK, C. M.; CHERRY, J. D.; CHRISTENSON, P.; LEWIS, K.; PINEDA, E.; SHLIAN, D.; DAWSON, J. A. \& BLUMBERG, D. A., 1992. A search for Bordetella pertussis infection in university students. Clinical Infectious Diseases, 14:464-471.
MOOI, F. R.; VAN LOO, I. H. \& KING, A. J., 2001. Adaptation of Bordetella pertussis to vaccination: A cause for its reemergence? Emerging Infectious Diseases, 7:526-528.

NTEZAYABO, B.; DE SERRES, G. \& DUVAL, B., 2003. Pertussis resurgence in Canada largely caused by a cohort effect. Pediatric Infectious Disease Journal, 22:22-27.

POSTELS-MULTANI, S.; SCHMITT, H. J.; WIRSINGVON KONIG, C. H.; BOCK, H. L. \& BOGAERTS, H., 1995. Symptoms and complications of pertussis in adults. Infection, 23:139-142.

SKOWRONSKI, D. M.; DE SERRES, G.; MacDONALD, D.; WU, W.; SHAW, C.; MacNABB, J.; CHAMPAGNE, S.; PATRICK, D. M. \& HALPERIN, S. A., 2002. The changing age and seasonal profile of pertussis in Canada. Journal of Infectious Diseases, 185:1448-1453.

STREBEL, P.; NORDIN, J.; EDWARDS, K.; HUNT, J.; BESSER, J.; BURNS, S.; AMUNDSON, G.; BAUGHMAN, A. \& WATTIGNEY, W., 2001. Populationbased incidence of pertussis among adolescents and adults, Minnesota, 1995-1996. Journal of Infectious Diseases, 183:1353-1359.

WALDMAN, E. A.; DA SILVA, L. J. \& MONTEIRO, C. A., 1999. Trajetória das doenças infecciosas: Da eliminação da poliomielite à reintrodução da cólera. Informe Epidemiológico do SUS, 8:5-47.

WHO (World Health Organization), 2001. WHO - Recommended Standards for Surveillance of Selected Vaccine-Preventable Diseases. 15 February 2002 $<$ http://www.who.int/vaccines-documents/DocsPDF01/pertussis.pdf $>$.

Recebido em 20 de março de 2003

Aprovado em 6 de maio de 2003 